\title{
Single-Photon Emission Mediated by Single-Electron Tunneling in Plasmonic Nanojunctions
}

\author{
Q. Schaeverbeke $\odot,{ }^{1,2}$ R. Avriller $\odot,{ }^{1}$ T. Frederiksen ${ }^{2,3},{ }^{2,}$ and F. Pistolesi ${ }^{1}$ \\ ${ }^{1}$ Univ. Bordeaux, CNRS, LOMA, UMR 5798, F-33405 Talence, France \\ ${ }^{2}$ Donostia International Physics Center (DIPC), E-20018 Donostia-San Sebastián, Spain \\ ${ }^{3}$ Ikerbasque, Basque Foundation for Science, E-48013 Bilbao, Spain
}

(Received 25 July 2019; published 12 December 2019)

\begin{abstract}
Recent scanning tunneling microscopy (STM) experiments reported single-molecule fluorescence induced by tunneling currents in the nanoplasmonic cavity formed by the STM tip and the substrate. The electric field of the cavity mode couples with the current-induced charge fluctuations of the molecule, allowing the excitation of photons. We investigate theoretically this system for the experimentally relevant limit of large damping rate $\kappa$ for the cavity mode and arbitrary coupling strength to a single-electronic level. We find that for bias voltages close to the first inelastic threshold of photon emission, the emitted light displays antibunching behavior with vanishing second-order photon correlation function. At the same time, the current and the intensity of emitted light display Franck-Condon steps at multiples of the cavity frequency $\omega_{c}$ with a width controlled by $\kappa$ rather than the temperature $T$. For large bias voltages, we predict strong photon bunching of the order of $\kappa / \Gamma$ where $\Gamma$ is the electronic tunneling rate. Our theory thus predicts that strong coupling to a single level allows current-driven nonclassical light emission.
\end{abstract}

DOI: 10.1103/PhysRevLett.123.246601

Electronic transport coupled to the field of an electromagnetic cavity can be realized in a wealth of different systems. This includes in the microwave range carbon nanotubes [1-5], quantum dots [6-9], and Josephson junctions [10-12], or in the optical range, molecules in plasmonic nanocavities formed by an STM tip with a substrate [13-24] and organic microcavities [25-27], or with waveguide quantum electrodynamic systems [28-30]. The reduction of the cavity volume $\mathcal{V}$ results in an increase of the zero-point quantum fluctuations of the electric field $E_{\mathrm{zpm}} \sim \mathcal{V}^{-1 / 2}$. This motivated optical studies of molecular two-level systems strongly coupled to the cavity field by the dipolar interaction $\Lambda_{d} \sim p E_{\mathrm{zpm}}$, (with $p$ the molecule dipole moment). One of the goals of this effort is to reach $\Lambda_{d}$ larger than $\kappa$, which has been and remains challenging, despite recent achievements [31]. On the other side, the coupling of a cavity mode to the current-induced charge fluctuations of a single-electronic level is given by a monopolar coupling constant $\Lambda_{m} \sim e L E_{\mathrm{zpm}}$ as derived in Ref. [32] (see also Ref. [33]), with $L$ the typical extension of the transport region and $e$ the electronic charge. Since typically in a given system $e L \gg p$, the monopolar coupling constant is much larger than the dipolar one [32].

Published by the American Physical Society under the terms of the Creative Commons Attribution 4.0 International license. Further distribution of this work must maintain attribution to the author(s) and the published article's title, journal citation, and DOI.
This probably contributed to the observation of values of $\Lambda_{m}$ larger than $\kappa$ in microwave cavities coupled to electronic transport $[4,6,8]$ and even approaching the cavity resonating frequency $\omega_{c}(\hbar=1)[11,39,40]$. Recent results in plasmonic cavities coupled to electronic transport $[17,21,22]$ thus open the possibility to explore transport through a single electronic level in these structures. This is expected to reach much larger coupling constants than those currently observed for purely dipolar coupling, requiring further theoretical investigations.

The system presents strong analogies with electron transport coupled to molecular vibrations. This has been investigated in different regimes, leading to the striking prediction of the Franck-Condon blockade [34,41,42] and its observation $[35,43]$. However, there are important differences. The first is the low quality factor of plasmonic cavities, which is typically of the order of 10 [31]. The second, and more interesting, is that the state of the optical or microwave cavity can be directly measured by detecting the emitted photons. It is thus important to investigate how transport through a molecule is linked to the property of the emitted radiation [44-46].

In this Letter we consider electronic transport through a single-level quantum dot, where the charge on the dot is coupled to the electric field of an electromagnetic cavity. We propose a theoretical model to obtain the current through the quantum dot taking into account the cavity dissipation $\kappa$, and arbitrary values of the coupling strength in the incoherent transport regime $\Gamma \ll k_{B} T$, with $\Gamma$ the electron tunneling rate, $T$ the temperature, and $k_{B}$ the 


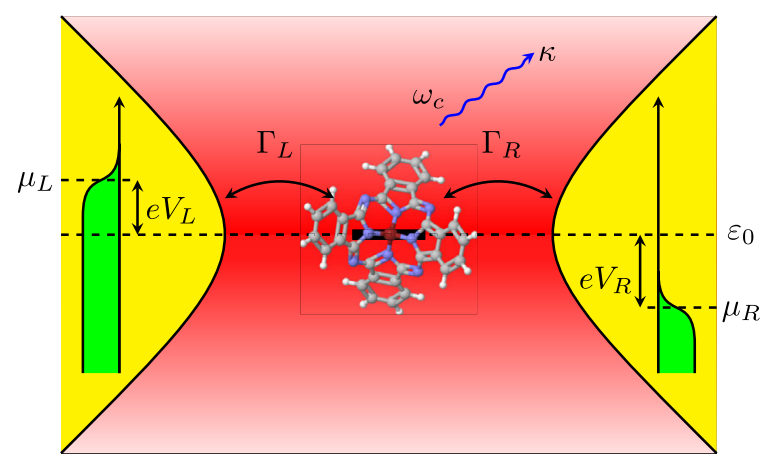

FIG. 1. Schematic of two metallic electrodes forming a plasmonic nanocavity characterized by a resonating frequency $\omega_{c} / 2 \pi$ and damping rate $\kappa$. A single electronic level $\varepsilon_{0}$ of a molecule in the nanogap couples to the electromagnetic radiation with coupling constant $\Lambda_{m}$. Electrons can tunnel to and from the dot with tunneling rates $\Gamma_{\alpha}$. Voltage drops, $V_{\alpha}$, with respect to $\varepsilon_{0}$ are indicated.

Boltzmann constant. Similarly to the Franck-Condon case, we find current steps at the inelastic thresholds for photon emission, but with a width controlled by $\kappa$ rather than $T$. We also derive the photon distribution and the second-order photon correlation function $g^{(2)}(t)$, where $t$ is the emission time. Its behavior for $t=0$ clearly shows that close to the first threshold for photon emission, for $\Gamma / \kappa \ll 1$, and $\Lambda_{m} \approx \omega_{c}$, photons antibunch: The junction becomes a single-photon source based on single-electron tunneling. This mechanism is different from the one assumed to be responsible for recently observed antibunching of emitted light in STM plasmonic nanojunctions involving multiple electronic levels [20]. For large bias voltages we find instead strong photon bunching with $g^{(2)}(0) \approx \kappa / \Gamma \gg 1$.

Model.-Figure 1 shows a schematic of the system at hand. The Hamiltonian is written $H=H_{S}+H_{I}+H_{B}$, where

$$
H_{S}=\tilde{\varepsilon}_{0} d^{\dagger} d+\omega_{c} a^{\dagger} a+\Lambda_{m} d^{\dagger} d\left(a+a^{\dagger}\right),
$$

with $d^{\dagger}$ the creation operator for the electron on the dot single level of energy $\tilde{\varepsilon}_{0}, a^{\dagger}$ the creation operator for the photon field, and $\Lambda_{m}=\lambda \omega_{c}$ the coupling constant. We follow Ref. [32] for the derivation of the interaction term [33]. We neglect direct coupling of the cavity field to the electrons in the leads, since this effect is analogous to the coupling of the cavity field to the photon bath. We treat the electrons in the leads and the propagating electromagnetic modes as a bath: $H_{B}=\sum_{\alpha k} \varepsilon_{\alpha k} c_{\alpha k}^{\dagger} c_{\alpha k}+\sum_{q} \omega_{q} b_{q}^{\dagger} b_{q}$, where $c_{\alpha k}^{\dagger}$ and $b_{q}^{\dagger}$ are the creation operators for the electrons on the leads $\alpha=L, R$ with energy $\varepsilon_{\alpha k}$ and for the propagating photons of energy $\omega_{q}$, respectively. The (linear) coupling to the bath is given by $H_{I}=$ $\sum_{\alpha k}\left[t_{\alpha k} c_{\alpha k}^{\dagger} d+t_{\alpha k}^{*} d^{\dagger} c_{\alpha k}\right]+\sum_{q}\left[l_{q} a^{\dagger} b_{q}+l_{q}^{*} b_{q}^{\dagger} a\right]$, with $t_{\alpha k}$ and $l_{q}$ the tunneling amplitudes. We first perform a standard
Lang-Firsov unitary transformation on the Hamiltonian $\tilde{H}=U H U^{\dagger}$, with $U=e^{\lambda d^{\dagger} d\left(a-a^{\dagger}\right)}$. This removes explicitly the electron-photon coupling term in $H_{S}$, shifts the dotlevel energy $\varepsilon_{0}=\tilde{\varepsilon}_{0}-\Lambda_{m}^{2} / \omega_{c}$ and modifies the $d$ operator in $H_{I}$ into $D=d e^{\lambda\left(a-a^{\dagger}\right)}$.

Master equation.-Let us define the reduced density matrix $\rho(t)$ for the $d$ and $a$ degrees of freedom after tracing out the bath. We assume that the molecule is sufficiently isolated from the substrate, as is reasonable for STM experiments performed on thin insulating films $[14,19$ $22]$. We consider then the relevant regime $\Gamma \ll k_{B} T \ll \omega_{c}$ where the dynamics of $\rho(t)$ can be described by the BornMarkov master equation

$$
\dot{\rho}(t)=\mathcal{L} \rho(t)=-i\left[\tilde{H}_{S}, \rho(t)\right]+\left(\mathcal{L}_{c}+\mathcal{L}_{e}\right) \rho(t) .
$$

The first term contributing to the Liouvilian operator $\mathcal{L}$ gives the coherent evolution of $\rho(t)$. The second one describes the damping of the cavity mode $[47,48]: \mathcal{L}_{c} \rho(t)=$ $\kappa\left(2 a \rho(t) a^{\dagger}-a^{\dagger} a \rho(t)-\rho(t) a^{\dagger} a\right) / 2-\kappa n_{B}\left[a^{\dagger},[a, \rho(t)]\right]$, with $n_{B}\left(\omega_{c}\right)=\left\{e^{\omega_{c} / k_{B} T}-1\right\}^{-1}$ the Bose distribution of photons in the bath at the cavity frequency. The last term describes incoherent electron tunneling $\mathcal{L}_{e} \rho(t)=\left[\mathcal{D}_{-} \rho(t)-\rho(t) \mathcal{D}_{+}\right.$, $\left.D^{\dagger}\right]+$ H.c. [49], where $\mathcal{D}_{ \pm}=\int_{-\infty}^{\infty} d \omega \int_{0}^{\infty} d t \Gamma_{\alpha}(\omega) f_{\alpha}^{ \pm}(\omega) e^{i \omega t}$ $D_{\mathrm{I}}(-t)$, and $\Gamma_{\alpha}=2 \pi \sum_{k}\left|t_{\alpha k}\right|^{2} \delta\left(\omega-\varepsilon_{\alpha k}\right)$ is the tunneling rate from the lead $\alpha$, which in the usual wide-band approximation becomes $\omega$ independent. Finally, $D_{\mathrm{I}}(-t)$ is the $D$ operator in the interaction representation with respect to $\tilde{H}_{S}$. We introduced the short-hand notation $f_{\alpha}^{+}(\omega)=1-f_{\alpha}^{-}(\omega)=n_{F}\left(\omega-\mu_{\alpha}\right)$, with $\mu_{\alpha}$ the chemical potential of lead $\alpha$ and $n_{F}(\omega)=\left\{e^{\omega / k_{B} T}+1\right\}^{-1}$ the Fermi distribution. The average or the correlation function of any observable $A, B$, can then be calculated in the stationary regime by $\langle A\rangle=\operatorname{Tr}\left[A \rho^{\mathrm{st}}\right]$ and $S_{A B}(t)=\langle A(t) B(0)\rangle=$ $\operatorname{Tr}\left[A e^{\mathcal{L} t} B \rho^{\mathrm{st}}\right][36,50]$, with $\rho^{\text {st }}$ the stationary solution of Eq. (2).

Electronic current.-Using the previous results, we derive the expression for the average electronic dc current evaluated at lead $\alpha$

$I_{\alpha}=\frac{e \Gamma_{\alpha}}{\pi} \operatorname{Re} \int_{-\infty}^{\infty} d \omega\left\{f_{\alpha}^{+}(\omega) S_{D D^{\dagger}}(\omega)+f_{\alpha}^{-}(\omega) S_{D^{\dagger} D}(\omega)\right\}$,

where we introduced the Fourier transform of $f_{\alpha}^{ \pm}, S_{D D^{\dagger}}$, and $S_{D^{\dagger} D}$. Equation (3) enables us to calculate the current in the presence of strong damping rates $\kappa$, that for plasmonic cavities reaches low quality factors $\omega_{c} / \kappa \approx 10$ [31]. It allows us to include the damping of the electromagnetic field during the tunneling process. This expression and $\rho^{\text {st }}$ can be evaluated numerically by projecting on the charge and harmonic oscillator basis.

Figure 2(a) reports the electronic current $I$ for strong coupling, $\lambda=1.4$, as a function of the relative voltage 
(a)

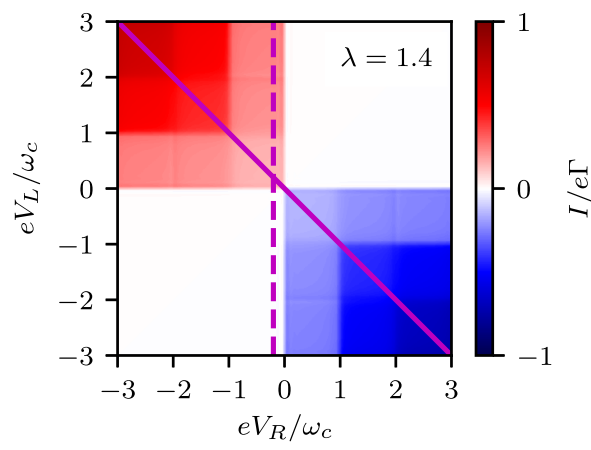

(b)

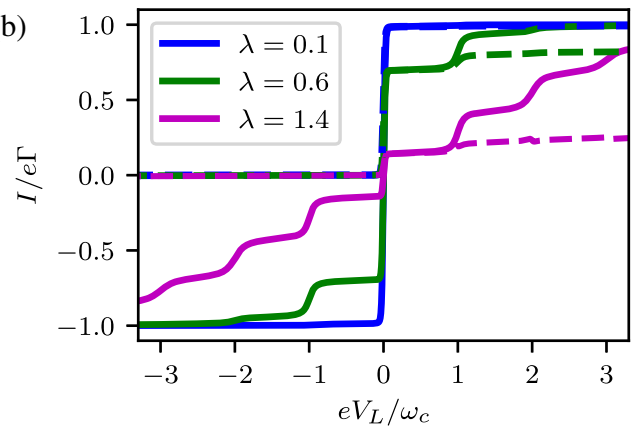

(c)

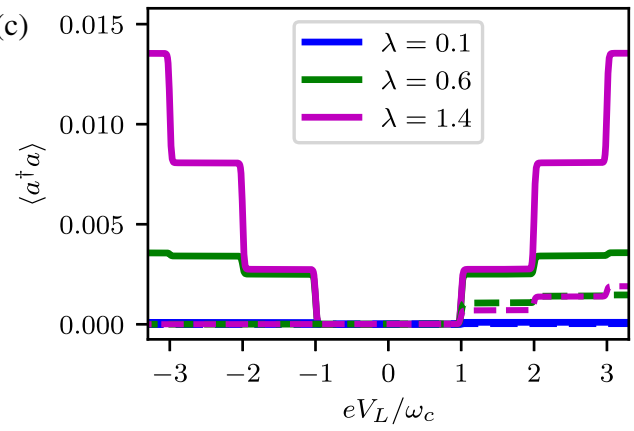

FIG. 2. (a) Electronic current $I$ vs the voltage drops $V_{L}$ and $V_{R}$ for $\lambda=1.4$. (b) Current-voltage characteristics and (c) average photon occupation $\left\langle a^{\dagger} a\right\rangle$ in the cavity corresponding to the voltage profiles indicated in panel (a) (dashed and full lines) for different electron-photon coupling strengths $\lambda=0.1$ (blue), $\lambda=$ 0.6 (green), and $\lambda=1.4$ (magenta). The model parameters are $\kappa=10 k_{B} T=0.1 \omega_{c}$ and $\Gamma_{L}=\Gamma_{R}=\Gamma / 2=10^{-3} \omega_{c}$.

drops $e V_{\alpha}=\mu_{\alpha}-\varepsilon_{0}$ between the chemical potential of lead $\alpha$ and the dot energy level [51]. Specific currentvoltage characteristics, corresponding to symmetric $\left(V_{L}=-V_{R}\right)$ and asymmetric $\left(e V_{R}=-0.2 \omega_{c}\right)$ voltage drops, are shown, respectively, as full and dashed lines in Fig. 2(b), for weak $(\lambda=0.1)$, moderate $(\lambda=0.6)$, and strong $(\lambda=1.4)$ coupling strengths. These exhibit similar features of the Franck-Condon blockade regime [34,41,42] with inelastic steps observed each time the voltage drop $e V_{L}=n \omega_{c}$ matches a multiple of the cavity-photon frequency. This is the threshold for one-electron tunneling while emitting $n$ photons in the cavity. The step heights are given by the Poisson distribution $\mathcal{P}_{n}(\lambda)=e^{-\lambda^{2}} \lambda^{2 n} / n$ ! and the width of the inelastic steps by the cavity-losses $\kappa$, which exceed the temperature broadening. This is analogous to the broadening of phonon sidebands by frictional damping [41], but our treatment is not bound to thermal equilibrium.

Emitted light.-We consider now the emitted light power $\sim \kappa \omega_{c}\left\langle a^{\dagger} a\right\rangle$, by plotting the average population of the cavity mode in Fig. 2(c) as a function of $V_{L}$. We find that the photon population also increases with bias voltage in a steplike manner [52], correlated to the evolution of the electronic current [49], thus confirming that single-electron tunneling is at the origin of light-emission inside the cavity. From Eq. (2), performing a secular approximation, we derive a rate equation for the photon population $P_{n}(t)$ [33]. A relevant experimental regime in plasmonic cavities is $\kappa \gg \Gamma$ and $\omega_{c} \gg k_{B} T$. Since the time between two tunneling events is much longer than the damping time of the cavity, typically the circulating photon leaks out before a new photon is emitted in the cavity. In this limit $P_{0} \approx 1$, and we find for the other populations $P_{n}=\Gamma_{0 n} / n \kappa \ll 1$, with $\Gamma_{0 n}=\sum_{\alpha} \Gamma_{\alpha} \mathcal{P}_{n}(\lambda) n_{F}\left(n \omega_{c}-\mu_{\alpha}\right)$ the cavity 0 to $n$ photons transition rate induced by a single tunneling event. The expression for $\left\langle a^{\dagger} a\right\rangle=\sum_{n} \Gamma_{0 n} / \kappa$ describes then accurately the emitted power.

Correlation function $g^{(2)}$. - In order to characterize the statistics of the emitted light, we compute the second-order correlation function $g^{(2)}(t)=\left\langle a^{\dagger} a^{\dagger}(t) a(t) a\right\rangle /\left\langle a^{\dagger} a\right\rangle^{2}$ [47, 53-55]. Let us begin with the $t=0$ case, $g^{(2)}(0)=$ $\left[\sum_{n} n(n-1) P_{n}\right] /\left(\sum_{n} n P_{n}\right)^{2}$. One can readily verify that in thermal equilibrium $g^{(2)}(0)=2$. On Fig. 3 we show $g^{(2)}(0)$ as a function of $V_{R}$ and $V_{L}$, for three different values of $\lambda$. As expected, for $e\left|V_{L}-V_{R}\right| \ll k_{B} T$, one always finds the value of 2 , corresponding to thermal equilibrium (pink regions on the diagonal $V_{L}=V_{R}$ ). Out of equilibrium we find either bunching $g^{(2)}(0)>1$ or antibunching $g^{(2)}(0)<1$. The antibunching appears for sufficiently strong coupling and is indicated by the blue regions with dashed border [where $g^{(2)}(0)=1$ ]. Treating both the electron tunneling and the thermal excitations as weak perturbation to the distribution $P_{n}=\delta_{n, 0}$ we obtain an analytical expression for $g^{(2)}(0)$ (see Ref. [33]) for voltages $e V_{L}=-e V_{R} \leq 2 \omega_{c}$.

The prediction of this expression agrees very well with the numerical calculations for $k_{B} T \geq 0.1 \omega_{c}$, [cf. thick lines in Figs. 4(a) and 4(b)]. At lower temperature we thus show only the result obtained with the analytical expression, that does not suffer from numerical instability. In the strong coupling regime, we predict a smooth crossover from the equilibrium value $g^{(2)}(0)=2$ at low voltage, to an antibunching $g^{(2)}(0)<1$ regime that appears close to the first inelastic threshold $\left(\left|e V_{L}\right| \approx \omega_{c}\right.$ or $\left|e V_{R}\right| \approx \omega_{c}$ ) [33,37]. As temperature decreases the region of antibunching expands, eventually including almost the full bias range $\left[0,2 \omega_{c}\right]$, cf. Fig. 4(b).

Figure 4(c) shows the minimum value taken by $g^{(2)}(0)$ when minimized on the plane $\left(V_{L}, V_{R}\right)$ for a given value of the coupling $\lambda$. One finds that antibunching can be observed 

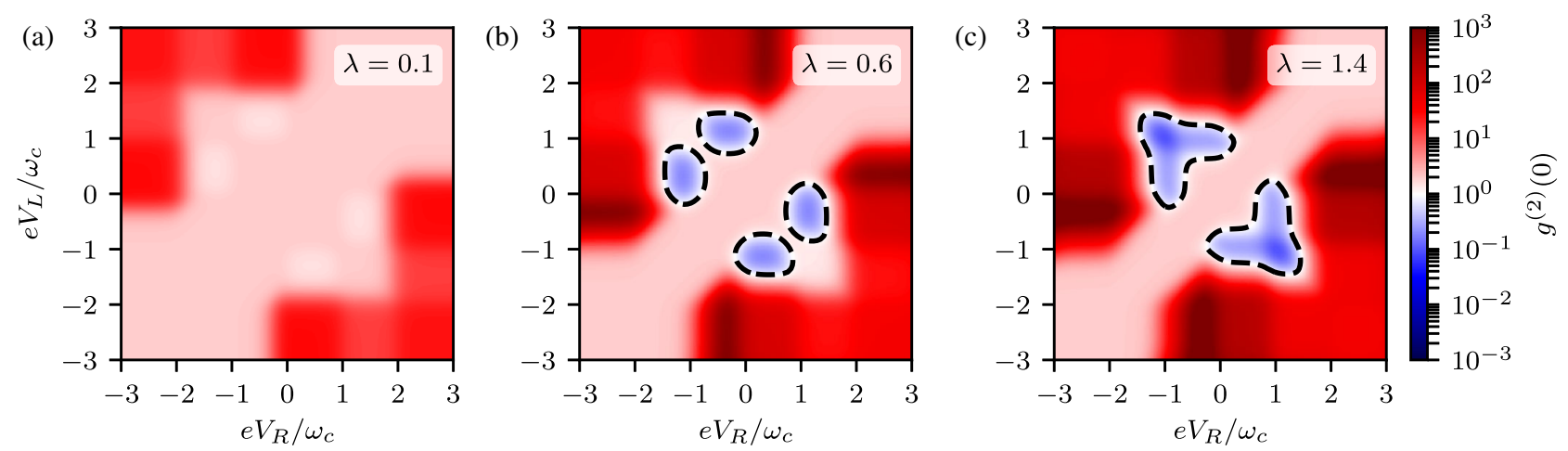

FIG. 3. Degree of coherence $g^{(2)}(0)$ for different electron-photon coupling strengths (a) $\lambda=0.1$, (b) $\lambda=0.6$, and (c) $\lambda=1.4$, respectively. The black dashed lines mark the contour $g^{(2)}(0)=1$ delimiting the regions of antibunching (blue areas). The model parameters are $\kappa=k_{B} T=0.1 \omega_{c}$ and $\Gamma_{L}=\Gamma_{R}=10^{-3} \omega_{c}$.
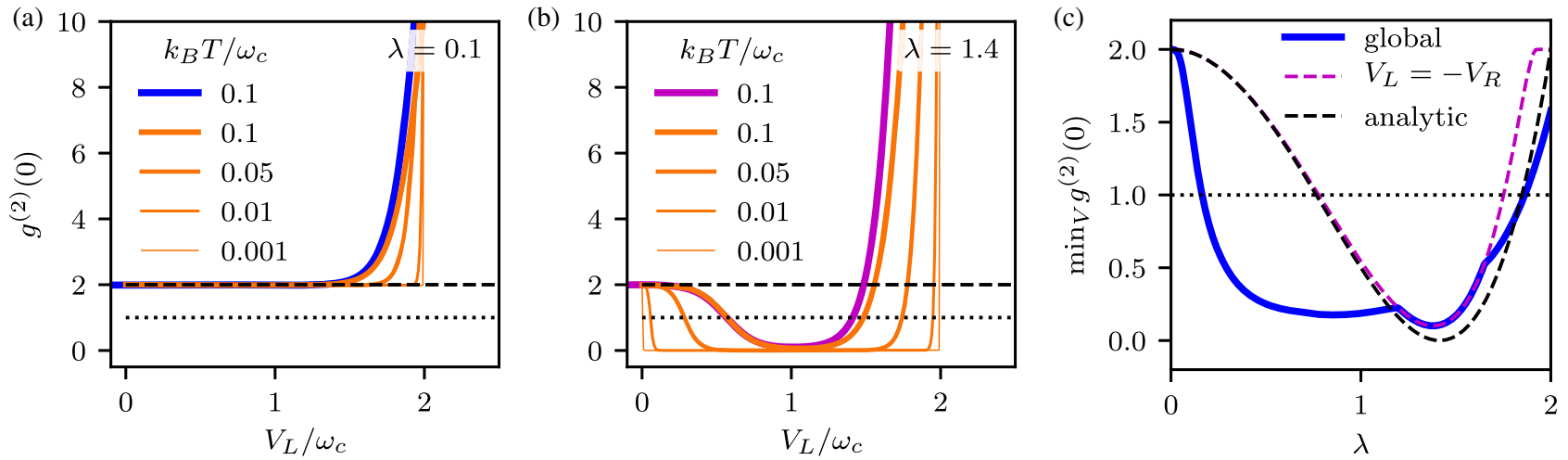

FIG. 4. (a), (b) Role of temperature $T$ on the degree of coherence $g^{(2)}(0)$ for $V_{L}=-V_{R}$ with (a) $\lambda=0.1$ and (b) $\lambda=1.4$. Blue and magenta thick lines are slices from Fig. 3(a,c), while the orange ones are computed from rate equations [33]. Dashed (dotted) horizontal line indicates $g^{(2)}(0)=2$ (1) for thermal (uncorrelated) photon emission. (c) Minimum value of $g^{(2)}(0)$ (thick blue line) on the plane $\left(V_{L}, V_{R}\right)$, for fixed $\lambda$ and $k_{B} T=0.1 \omega_{c}$. Dashed magenta line shows that minimum constrained to $V_{L}=-V_{R}$. Dashed black line gives the $T=0$ analytical prediction $\left(\lambda^{2}-2\right)^{2} / 2$. Other parameters are those of Fig. 3 .

for $\lambda>0.17$ for an asymmetric bias configuration, thus being at reach of present experiments. The case of symmetric bias is also shown, with antibunching beginning at $\lambda>0.76$. Let us discuss now the antibunching mechanism for symmetric bias. When $P_{n+1} \ll P_{n}$, at lowest order $g^{(2)}(0)=2 P_{2} / P_{1}^{2}$. This expression gives 2 for thermal distribution $P_{n} \sim e^{-n \omega_{c} / k_{B} T}$. Antibunching is thus achieved for $2 P_{2} \ll P_{1}^{2}$. At very low temperature the only way to populate the state 2 is either a two-photons transition (for $\left.e V_{L}>2 \omega_{c}\right)$, or an electron-tunneling assisted transition from the state 1 to the state 2 , controlled by $\Gamma_{12}=$ $e^{-\lambda^{2}} \lambda^{2}\left(2-\lambda^{2}\right)^{2} \sum_{\alpha} \Gamma_{\alpha} n_{F}\left(\omega_{c}-\mu_{\alpha}\right) / 2$ (for $e V_{L}>\omega_{c}$ ). As a confirmation one finds that $P_{1} \approx P_{0} \Gamma_{01} / \kappa$ and $P_{2} \approx P_{1} \Gamma_{12} / 2 \kappa$, testifying that the result is just due to a balance between the light leaked out of the cavity and the photons emitted in the cavity by the tunneling electrons. From the explicit expression of $\Gamma_{12}$ at low temperature $\left(k_{B} T \ll \omega_{c}\right)$ one then finds that the minimum value of $g^{(2)}(0)$ is approximated by $\left(\lambda^{2}-2\right)^{2} / 2$ [dashed black line in Fig. 4(c)], tracing the $\lambda$ dependence of $\Gamma_{12}$. Its vanishing for $\lambda=\sqrt{2}$ is thus at the origin of the antibunching. A similar effect has also been recently reported in a dc-biased Josephson junction coupled to microwave resonators [11,12].

For larger voltages $e V_{L} \geq 2 \omega_{c}$, we obtain analytically [33] $g^{(2)}(0) \approx \kappa / 2 \Gamma$, as predicted by the numerical simulations giving a smooth evolution to strong bunching. This result agrees with the infinite bias voltage limit result recently reported in Ref. [56]. Finally, the time dependence of $g^{(2)}(t)$ as obtained by the numerical calculations shows a smooth crossover on a timescale $1 / \kappa$ from $g^{(2)}(0)=2$ to $g^{(2)}(\infty)=1$ (uncorrelated photons) [54]. Only for $\lambda \approx 1$ we observe weak oscillations [33].

Conclusions. - Charge fluctuations induced by electronic transport in a molecular single-electronic level are expected to couple strongly to the plasmonic mode formed by an STM tip and the substrate. We derived an expression for the current taking into account the strong damping of 
these cavities and obtained the current, emitted light intensity, and the correlation function $g^{(2)}(t)$. We showed that when the coupling strength is of the order $\lambda \sim 1$, Franck-Condon steps appear in both the current and the light intensity. Nonclassical light can be emitted for a coupling strength in the range $0.17<\lambda<1.8$ for bias voltage near the 1 photon emission threshold. This prediction can be relevant for a series of experiments on STM cavities [16-24]. The importance of single-level descriptions has further been reinforced experimentally after our initial submission [57]. The fast evolution of the cavity $\left(\kappa>k_{B} T\right)$ might question the validity of the Markov approximation. We estimate that the non-Markovian contributions are negligible as far as $k_{B} T \ll \omega_{c}$, but investigating low-frequency response of plasmonic cavities could unravel such effects. Another interesting direction is the study of current response in the presence of light irradiation of the plasmonic junction. Finally, we note that a local quantum emitter can be used to sense the environment of the molecule with the minimal quantum of energy.

We thank Tomáš Neuman, Javier Aizpurua, and Guillaume Schull for stimulating discussions. This work was supported by IDEX Bordeaux (No. ANR-10-IDEX03-02) and Euskampus Transnational Common Laboratory QuantumChemPhys. T. F. acknowledges Grant No. FIS 2017-83780-P from the Spanish Ministerio de Economía y Competitividad. R. A. acknowledges financial support by the Agence Nationale de la Recherche project CERCa, ANR-18-CE30-0006.

[1] M. R. Delbecq, V. Schmitt, F. D. Parmentier, N. Roch, J. J. Viennot, G. Fève, B. Huard, C. Mora, A. Cottet, and T. Kontos, Phys. Rev. Lett. 107, 256804 (2011).

[2] L. E. Bruhat, J. J. Viennot, M. C. Dartiailh, M. M. Desjardins, T. Kontos, and A. Cottet, Phys. Rev. X 6, 021014 (2016).

[3] A. Cottet, M. C. Dartiailh, M. M. Desjardins, T. Cubaynes, L. C. Contamin, M. Delbecq, J. J. Viennot, L. E. Bruhat, B. Douot, and T. Kontos, J. Phys. Condens. Matter 29, 433002 (2017).

[4] L. E. Bruhat, T. Cubaynes, J. J. Viennot, M. C. Dartiailh, M. M. Desjardins, A. Cottet, and T. Kontos, Phys. Rev. B 98, 155313 (2018).

[5] T. Cubaynes, M. R. Delbecq, M. C. Dartiailh, R. Assouly, M. M. Desjardins, L. C. Contamin, L. E. Bruhat, Z. Leghtas, F. Mallet, A. Cottet, and T. Kontos, npj Quantum Inf. 5, 47 (2019).

[6] X. Mi, J. V. Cady, D. M. Zajac, P. W. Deelman, and J. R. Petta, Science 355, 156 (2017).

[7] X. Mi, J. V. Cady, D. M. Zajac, J. Stehlik, L. F. Edge, and J. R. Petta, Appl. Phys. Lett. 110, 043502 (2017).

[8] A. Stockklauser, P. Scarlino, J. V. Koski, S. Gasparinetti, C. K. Andersen, C. Reichl, W. Wegscheider, T. Ihn, K. Ensslin, and A. Wallraff, Phys. Rev. X 7, 011030 (2017).

[9] Y.-Y. Liu, K. D. Petersson, J. Stehlik, J. M. Taylor, and J. R. Petta, Phys. Rev. Lett. 113, 036801 (2014).
[10] A. Wallraff, D. I. Schuster, A. Blais, L. Frunzio, R.-S. Huang, J. Majer, S. Kumar, S. M. Girvin, and R. J. Schoelkopf, Nature (London) 431, 162 (2004).

[11] C. Rolland, A. Peugeot, S. Dambach, M. Westig, B. Kubala, Y. Mukharsky, C. Altimiras, H. le Sueur, P. Joyez, D. Vion, P. Roche, D. Esteve, J. Ankerhold, and F. Portier, Phys. Rev. Lett. 122, 186804 (2019).

[12] A. Grimm, F. Blanchet, R. Albert, J. Leppäkangas, S. Jebari, D. Hazra, F. Gustavo, J.-L. Thomassin, E. Dupont-Ferrier, F. Portier, and M. Hofheinz, Phys. Rev. X 9, 021016 (2019).

[13] R. Berndt, J. K. Gimzewski, and P. Johansson, Phys. Rev. Lett. 67, 3796 (1991).

[14] X. H. Qiu, G. V. Nazin, and W. Ho, Science 299, 542 (2003).

[15] N. L. Schneider and R. Berndt, Phys. Rev. B 86, 035445 (2012).

[16] R. Zhang, Y. Zhang, Z. C. Dong, S. Jiang, C. Zhang, L. G. Chen, L. Zhang, Y. Liao, J. Aizpurua, Y. Luo, J. L. Yang, and J. G. Hou, Nature (London) 498, 82 (2013).

[17] G. Reecht, F. Scheurer, V. Speisser, Y. J. Dappe, F. Mathevet, and G. Schull, Phys. Rev. Lett. 112, 047403 (2014).

[18] P. Merino, C. Große, A. Roslawska, K. Kuhnke, and K. Kern, Nat. Commun. 6, 8461 (2015).

[19] Y. Zhang, Y. Luo, Y. Zhang, Y.-J. Yu, Y.-M. Kuang, L. Zhang, Q.-S. Meng, Y. Luo, J.-L. Yang, Z.-C. Dong, and J. G. Hou, Nature (London) 531, 623 (2016).

[20] L. Zhang, Y.-J. Yu, L.-G. Chen, Y. Luo, B. Yang, F.-F. Kong, G. Chen, Y. Zhang, Q. Zhang, Y. Luo, J.-L. Yang, Z.-C. Dong, and J. G. Hou, Nat. Commun. 8, 580 (2017).

[21] H. Imada, K. Miwa, M. Imai-Imada, S. Kawahara, K. Kimura, and Y. Kim, Phys. Rev. Lett. 119, 013901 (2017).

[22] B. Doppagne, M. C. Chong, H. Bulou, A. Boeglin, F. Scheurer, and G. Schull, Science 361, 251 (2018).

[23] M. C. Chong, N. Afshar-Imani, F. Scheurer, C. Cardoso, A. Ferretti, D. Prezzi, and G. Schull, Nano Lett. 18, 175 (2018).

[24] T. Neuman, R. Esteban, D. Casanova, F. J. Garcia-Vidal, and J. Aizpurua, Nano Lett. 18, 2358 (2018).

[25] E. Orgiu, J. George, J. A. Hutchison, E. Devaux, J. F. Dayen, B. Doudin, F. Stellacci, C. Genet, J. Schachenmayer, C. Genes, G. Pupillo, P. Samorì, and T. W. Ebbesen, Nat. Mater. 14, 1123 (2015).

[26] T. Schwartz, J. A. Hutchison, C. Genet, and T. W. Ebbesen, Phys. Rev. Lett. 106, 196405 (2011).

[27] D. Hagenmüller, J. Schachenmayer, S. Schütz, C. Genes, and G. Pupillo, Phys. Rev. Lett. 119, 223601 (2017).

[28] Z. Chen, Y. Zhou, and J.-T. Shen, Opt. Lett. 41, 3313 (2016).

[29] Z. Chen, Y. Zhou, and J.-T. Shen, Phys. Rev. A 96, 053805 (2017).

[30] Y. Zhou, Z. Chen, L. V. Wang, and J.-T. Shen, Opt. Lett. 44, 475 (2019).

[31] R. Chikkaraddy, B. de Nijs, F. Benz, S. J. Barrow, O. A. Scherman, E. Rosta, A. Demetriadou, P. Fox, O. Hess, and J. J. Baumberg, Nature (London) 535, 127 (2016).

[32] A. Cottet, T. Kontos, and B. Douçot, Phys. Rev. B 91, 205417 (2015).

[33] See Supplemental Material at http://link.aps.org/ supplemental/10.1103/PhysRevLett.123.246601 for the detailed derivation about the electron-photon coupling, the 
dependence of the current on the plasmon-molecule coupling strength and a more detailed investigation of the second-order correlation function of the plasmon field, which includes Refs. [32,34-38].

[34] J. Koch and F. von Oppen, Phys. Rev. Lett. 94, 206804 (2005).

[35] E. Burzurí, Y. Yamamoto, M. Warnock, X. Zhong, K. Park, A. Cornia, and H. S. van der Zant, Nano Lett. 14, 3191 (2014).

[36] C. Cohen-Tannoudji, J. Dupont-Roc, and G. Grynberg, Atom-Photon Interactions: Basic Processes and Applications (Wiley, Hoboken, 1998).

[37] X. T. Zou and L. Mandel, Phys. Rev. A 41, 475 (1990).

[38] F. Pistolesi and S. Labarthe, Phys. Rev. B 76, 165317 (2007).

[39] C. Altimiras, O. Parlavecchio, P. Joyez, D. Vion, P. Roche, D. Esteve, and F. Portier, Appl. Phys. Lett. 103, 212601 (2013).

[40] M. C. Cassidy, A. Bruno, S. Rubbert, M. Irfan, J. Kammhuber, R. N. Schouten, A. R. Akhmerov, and L. P. Kouwenhoven, Science 355, 939 (2017).

[41] S. Braig and K. Flensberg, Phys. Rev. B 68, 205324 (2003).

[42] J. Koch, F. von Oppen, and A. V. Andreev, Phys. Rev. B 74, 205438 (2006).

[43] R. Leturcq, C. Stampfer, K. Inderbitzin, L. Durrer, C. Hierold, E. Mariani, M. G. Schultz, F. von Oppen, and K. Ensslin, Nat. Phys. 5, 327 (2009).

[44] M. Galperin and A. Nitzan, Phys. Rev. Lett. 95, 206802 (2005).
[45] K. Kaasbjerg and A. Nitzan, Phys. Rev. Lett. 114, 126803 (2015).

[46] F. Xu, C. Holmqvist, G. Rastelli, and W. Belzig, Phys. Rev. B 94, 245111 (2016).

[47] W. H. Louisell, Quantum Statistical Properties of Radiation (Wiley, Hoboken, 1973).

[48] C. W. Gardiner and M. J. Collett, Phys. Rev. A 31, 3761 (1985).

[49] R. Avriller, B. Murr, and F. Pistolesi, Phys. Rev. B 97, 155414 (2018).

[50] P. G. Kirton, A. D. Armour, M. Houzet, and F. Pistolesi, Phys. Rev. B 86, 081305(R) (2012).

[51] We plot the symmetrized current $I=\left(I_{L}+I_{R}\right) / 2$, since for asymmetric bias voltages one observes in some cases weak nonconserving contributions.

[52] At this level of approximation, the steps of the cavity-mode occupation appear to be broadened on the scale given by temperature instead of $\kappa$.

[53] D. F. Walls and G. J. Milburn, Quantum Optics, 2nd ed. (Springer, Berlin, 2008).

[54] P. R. Rice and H. J. Carmichael, IEEE J. Quantum Electron. 24, 1351 (1988).

[55] R. J. Glauber, Phys. Rev. 131, 2766 (1963).

[56] T. L. van den Berg and P. Samuelsson, Phys. Rev. B 100, 035408 (2019).

[57] C. C. Leon, O. Gunnarsson, D. G. de Oteyza, A. Rosławska, P. Merino, A. Grewal, K. Kuhnke, and K. Kern, arXiv: 1909.08117 